\title{
In vitro three-dimensional organotypic culture models of the oral mucosa
}

\author{
Mitchell Klausner $^{1}$ (D) $\cdot$ Yuki Handa $^{2} \cdot$ Seiya Aizawa ${ }^{3}$
}

Received: 25 June 2020 / Accepted: 14 December 2020 / Published online: 14 January 2021 / Editor: Tetsuji Okamoto

(C) The Society for In Vitro Biology 2021

\begin{abstract}
Three-dimensional, organotypic models of the oral mucosa have been developed to study a wide variety of phenomena occurring in the oral cavity. Although a number of models have been developed in academic research labs, only a few models have been commercialized. Models from academic groups offer a broader range of phenotypes while the commercial models are more focused on the oral and gingival mucosa. The commercialized models are manufactured under highly controlled conditions and meet the requirements of quality standards, which leads to high levels of reproducibility. These in vitro models have been used to evaluate the irritancy of oral care products such as toothpastes, mouthwashes, and mucoadhesives. The effects of cigarette smoke on oral cavity tissues have been studied and compared to those of e-cigarettes. Oral tissue models have facilitated investigation of the mechanisms of oral mucositis and oral candidiasis and have been used to examine transbuccal drug delivery rates and the absorption of nanoparticles. Infection studies have investigated the effects of HIV-1 along with the effects of commensal and pathogenic bacteria. More recently, a differentiated oral tissue model has been shown to express the ACE2 receptor, which is known to be important for the receptor-mediated entry of the SARS-CoV-2 coronavirus into human cells and tissues. Hence, oral mucosal models may find application in determining whether viral infection of the oral mucosa is possible and whether such infection has implications vis-a-vis the current COVID-19 pandemic. As is apparent, these models are used in a broad variety of applications and often offer advantages versus animal models in terms of reproducibility, avoiding species extrapolation, and the ethical concerns related to human and animal experimentation. The goals of this paper are to review commercially available models of the human buccal and gingival mucosa and highlight their use to gain a better understanding of a broad range of phenomena affecting tissues in the oral cavity.
\end{abstract}

Keywords Oral mucosal model $\cdot$ Buccal tissue model $\cdot$ Gingival tissue model $\cdot$ Organotypic tissues

\section{Introduction}

Three-dimensional (3D), organotypic tissue models offer several advantages over cells in monolayer (2D culture) since in many aspects the tissue models replicate the differentiated structure and function of native tissues (Jensen and Teng 2020). In native buccal and gingival tissues, the epithelial barrier prevents or limits the passage of toxins, microbes,

Mitchell Klausner

mklausner@mattek.com

1 MatTek Corporation, 200 Homer Avenue, Ashland, MA 01721, USA

2 Kurabo Industries Ltd, Neyagawa, Osaka, Japan

3 LION Corporation, Odawara, Kanagawa, Japan and chemicals into the underlying basal layers of the tissue where they could damage the proliferating basal stem cells (Bierbaumer et al. 2018). Likewise, the barrier of these tissues prevents passage of xenobiotics into the vasculature where they could pose systemic toxicity problems (Komiyama et al. 2019). In monolayer culture models, xenobiotics have direct access to the proliferative cells, and thus, they cannot appropriately model exposure risk (Moharamzadeh et al. 2008; Moghaddam et al. 2016). Another advantage of the organotypic tissue models relates to route of exposure to test materials and/or xenobiotics. The 3D oral and gingival tissue models are cultured at the air-liquid interface (ALI) in cell culture inserts (e.g., Millicell ${ }^{\mathrm{TM}}$ or Transwell ${ }^{\mathrm{TM}}$ ) with a microporous membrane bottom. In ALI culture, cells are seeded into the inserts, and after a period of submerged culture, the culture medium is removed from the apical culture surface. At the ALI, the cells are fed solely through the microporous 
membrane underneath the growing tissue, and the apical surface of that tissue is exposed to the atmosphere within the incubator. Thus, culture at the ALI allows for clinically relevant exposure of test articles or xenobiotics since these materials can be applied directly to the apical tissue surface (Klausner et al. 2007). In contrast, monolayer cultures are submerged in aqueous medium and test articles need to be dissolved prior to application to the cells. Culture at the ALI also induces differentiation so that tissue properties more closely reproduce in vivo characteristics (Delcourt-Huard et al. 1997). Sensitivity to drugs and drug metabolism, gene and protein expression, cell-to-cell communication, and other phenomena are more accurately represented in 3D cultures than in 2D systems, as recently reviewed (Jensen and Teng 2020). The 3D organotypic tissue models of the oral mucosa are more accessible and available than the animal models traditionally used to study the oral cavity, which include monkey, rat, mouse, dog, pig, rabbit, cavy, sheep, and buffalo (Samaranayake and Samaranayake 2001; Sa et al. 2016). Additionally, toothpastes and other oral care products fall under legislation applicable to cosmetics which includes products for use "with the teeth and the mucous membranes of the oral cavity ...cleaning them, perfuming them, changing their appearance, protecting them, keeping them in good condition...." This legislation prohibits animal testing of oral care products in the European Union (EU Regulation EC No 1223/2009) and many non-EU oral care companies have also committed to reducing or eliminating the use of animal models for the testing of their products. Inter-species differences between animals and humans can make extrapolation of animal data difficult, whereas the 3D culture models, which comprised human cells, avoid this issue. Additionally, because the oral tissue models are less complex than the whole organism models, researchers can isolate specific phenomena of interest and can more easily interpret experimental data. Due to these advantages, oral tissue models cultured using human cells are often the test system of choice for many applications (https://www.episkin.com/HGE-GingivalEpithelium).

\section{Models of Oral Mucosal Tissue}

The oral mucosa of the human oral cavity can be broken in three categories: (1) the lining (buccal, sublingual, soft palate tissues), (2) masticatory (gingival and hard palate tissues), and (3) specialized mucosa (dorsal surface of the tongue). In vivo, all of these tissue subtypes consist of an outer, stratified squamous epithelium comprising oral keratinocytes and an underlying collagen-based connective tissue (referred to as the lamina propria) which contains fibroblasts, along with blood vessels, nerve endings, and salivary glands in its lower layers. The lining mucosa is $\sim 60 \%$ of the total surface area of the oral cavity and the epithelial layer remains non-cornified. The masticatory tissue represents $\sim 25 \%$ of the oral cavity and is cornified and the specialized mucosa of the tongue is $\sim 15 \%$ with interspersed cornified and non-cornified regions (Squier and Kremer 2001). Many in vitro models reflecting the different phenotypes of the various oral mucosal tissues have been developed, as comprehensively reviewed (Bierbaumer et al. 2018).

This review will focus on commercially available models and the various applications for which they have been utilized. Currently, four tissue-engineered oral mucosa models are commercially available, the SkinEthic ${ }^{\mathrm{TM}}$ Human Oral Epithelium (HOE) and the SkinEthic Human Gingival Epithelium (HGE) constructs from EPISKIN (Lyon, France), and the EpiOral ${ }^{\mathrm{TM}}$ and EpiGingival ${ }^{\mathrm{TM}}$ tissues from MatTek Corporation (Ashland, MA). The SkinEthic tissues are produced following the ISO 9001: 2015 quality standards and the MatTek tissues are manufactured in accordance with the Good Manufacturing Practice (GMP) quality standards. These standards require that each lot of tissue meets preestablished lot release criteria which enhances the reproducibility of these systems. The commercial tissues are produced on a regular basis and are shipped to researchers throughout the world (https://www.episkin.com/HOE-Oral-Epithelium).

MatTek Corporation has produced an oral mucosal model, EpiOral, with a buccal phenotype since 2006. The tissue mod$\mathrm{el}$ is cultured in serum-free medium in cell culture inserts with a microporous membrane bottom ( $0.4 \mu \mathrm{m}$ average pore size), at the air-liquid interface (i.e., the apical tissue surface is exposed to the atmosphere as opposed to being submerged in culture medium; see Fig. 1). Normal human oral keratinocytes harvested from non-diseased donor tissues (either from

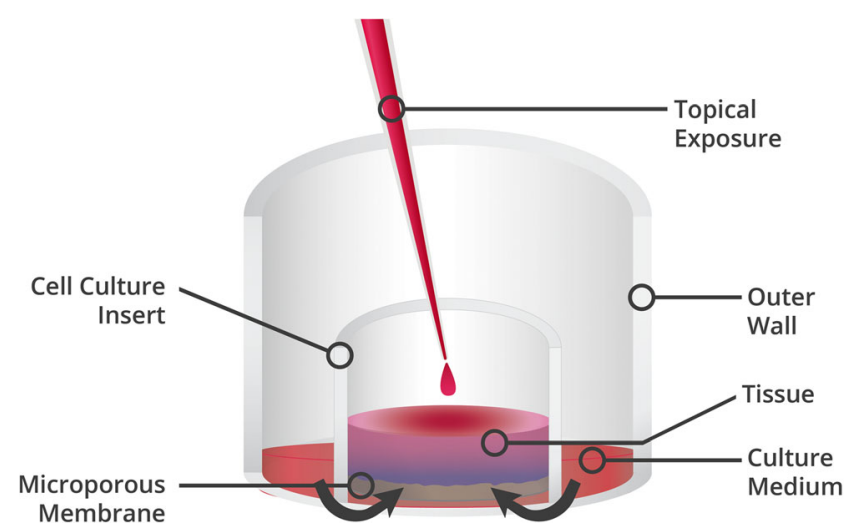

Figure 1. Schematic of culture at the air-liquid interface (ALI). Cells are seeded into the cell culture inserts $(\mathrm{CCI})$ onto the microporous membrane (which is typically coated with an extracellular matrix protein such as collagen). After a brief period of submerged culture in which culture medium is placed beneath and into the CCI, the medium is removed from the CCI so that the apical tissue surface is exposed to the atmosphere. Feeding of the tissue continues exclusively by medium passing through the microporous membrane until the tissue is fully differentiated. Since the apical surface of the fully differentiated tissue is not submerged, neat test articles can be applied directly to the tissue without first diluting them in an aqueous medium. 
cadavers or from patients undergoing tooth extractions) are utilized. The EpiOral tissue is non-cornified with limited barrier lipid content (Klausner et al. 2007). Transepithelial electrical resistance (TEER), a non-invasive, quantitative method to assess the barrier integrity of epithelial tissues (Benson et al. 2013), has been used to characterize the tissues. Average TEER of $413 \pm 138 \Omega * \mathrm{~cm}^{2}(n=22)$ has been reported for the EpiOral tissue model (Klausner et al. 2007). EpiOral tissues express cytokeratins 13 and 14 along with innate immune markers such as human beta defensin 1 (HBD1) and HBD3 and toll-like receptor 2 (TLR2) and TLR4, similar to native buccal tissue (Kimball et al. 2006). In addition to EpiOral, MatTek produces an oral tissue with a gingival phenotype, EpiGingival, which is cornified and has higher barrier lipid content and a more robust barrier than the EpiOral tissue, as confirmed by average TEER of $516 \pm 122 \Omega^{*} \mathrm{~cm}^{2}(n=10, p=$ 0.048 vs. the EpiOral tissue). EpiGingival tissues express cytokeratin 13 in the apical tissue layers and cytokeratin 14 in the basal tissue layers and are much more resilient to damage by surfactants such as Triton X-100 (Klausner et al. 2007). Hematoxylin and eosin (H\&E)-stained cross-sections of the EpiOral and EpiGingival tissues are shown in Fig. 2. Both tissues have actively dividing cells, as evidenced by Ki67 staining in the basal layer of the tissue, similar to native tissue (Yadev et al. 2011; Schlage et al. 2014). MatTek also produces full thickness versions of the EpiOral and EpiGingival tissues, EpiOral-FT and EpiGingival-FT, which include an underlying lamina propria consisting of a collagen matrix containing normal human buccal or gingival fibroblasts harvested from healthy explant tissue (Klausner et al. 2007; Morse et al. 2018). Finally, MatTek has incorporated dendritic cells into the full thickness EpiOral tissue (Schlage et al. 2014), although the functionality of the dendritic cells within the tissue has not been investigated in a rigorous manner.
The SkinEthic-reconstructed human oral epithelial (HOE) model is cultured using TR146 cells which were derived from a squamous cell carcinoma of the buccal mucosa (Rupniak et al. 1985). The HOE is cultivated on an inert polycarbonate filter at the air-liquid interface in a chemically defined medium. This model forms an epithelial tissue devoid of stratum corneum and histologically resembles the mucosa of the oral cavity. The HOE expresses cytokeratins 6 and 16, along with CD44 (https://www.episkin.com/HOE-Oral-Epithelium) and TEER was reported to be $55-122 \Omega * \mathrm{~cm}^{2}$ (Jacobsen et al. 1995). The SkinEthic Human Gingival Epithelium (HGE) model is produced by culturing normal human gingival cells on an inert polycarbonate filter at the air-liquid interface in a chemically defined medium. This model is histologically similar to the outer cell layers of the human gum tissue and expresses cytokeratins 10 and 13 and filaggrin (https://www. episkin.com/HGE-Gingival-Epithelium). In one study, the HGE model was cultured in conjunction with immunological cells (Brown et al. 2019). Both the HOE and HGE models contain proliferating (Ki-67 positive) basal cells but the HOE also contains $\mathrm{Ki}-67$ positive cells in the upper layers of the mucosal model, while in normal mucosa, proliferating cells are restricted to the basal layer (Yadev et al. 2011). H\&E-stained cross-sections of the HOE and HGE tissues are shown in Fig. 3.

\section{Quality Control of the EpiOral Tissue Model}

The commercial tissue models are produced under highly controlled manufacturing conditions which improve their reproducibility. MatTek's EpiOral (ORL-200) and EpiGingival (GIN100) tissues are manufactured under Good Manufacturing Procedures (GMP) and each lot is evaluated prior to shipping,

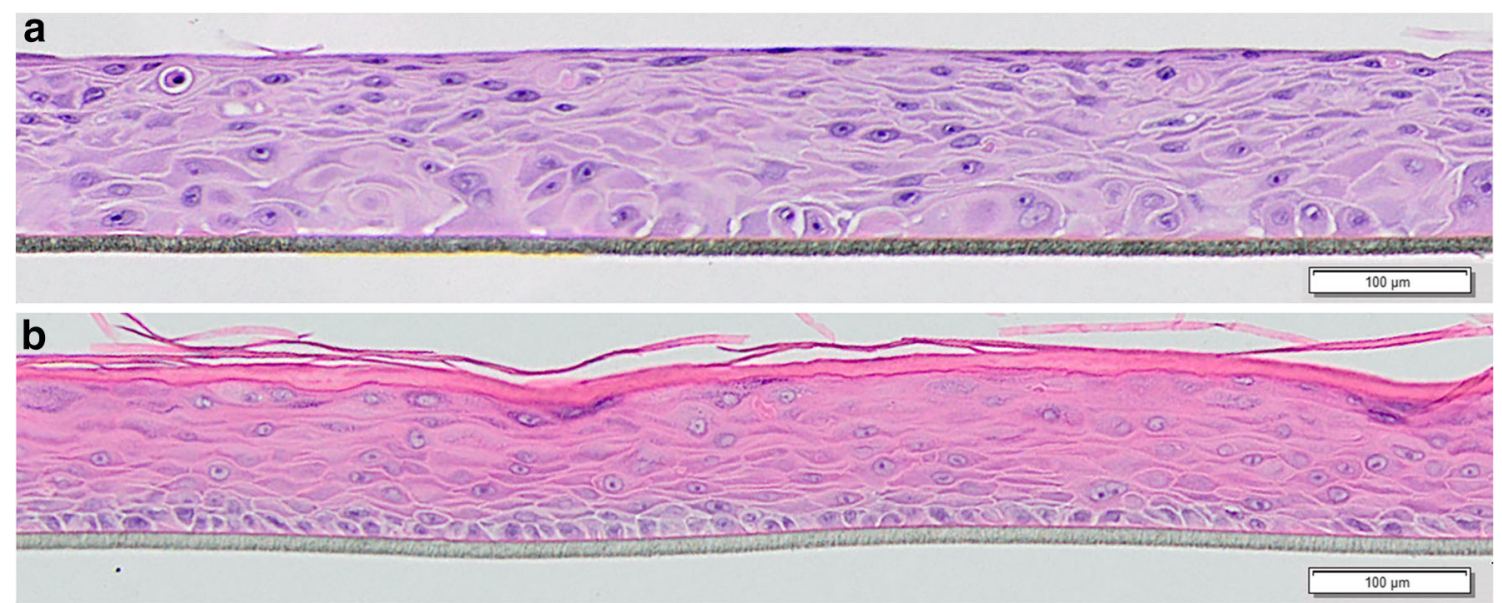

Figure 2. Hematoxylin and eosin (H\&E)-stained cross-sections of the MatTek organotypic oral mucosa tissue models: (a) EpiOral (ORL-200) and (b) EpiGingival (GIN-100). 


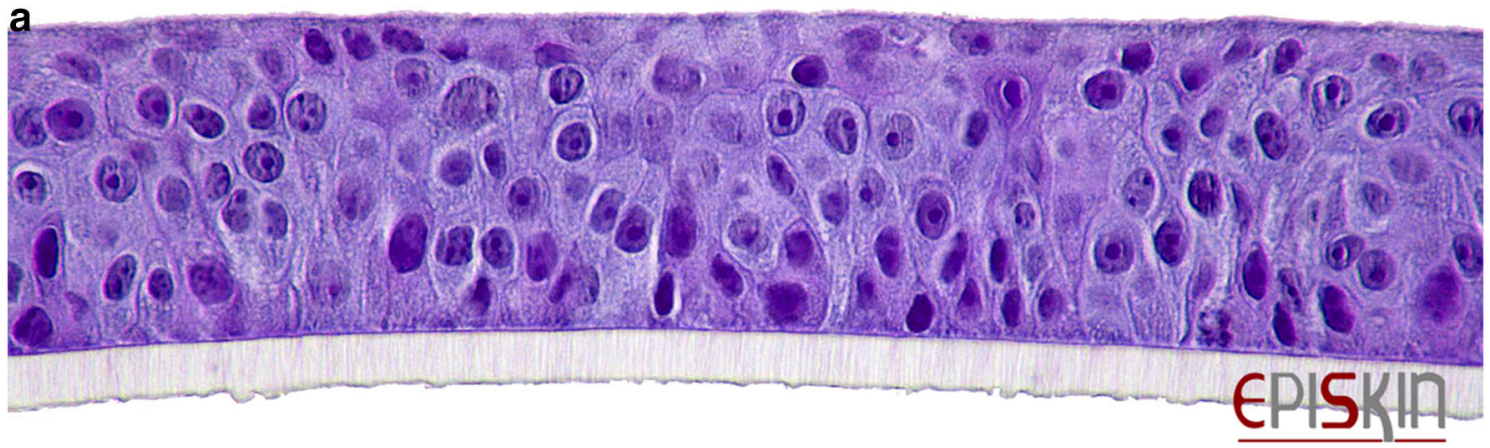

b



Figure 3. Hematoxylin and eosin (H\&E)-stained cross-sections of the SkinEthic organotypic oral mucosa tissue models: (a) human oral epithelial (HOE) and (b) Human Gingival Epithelium (HGE).

and after storage for $24 \mathrm{~h}$ storage at $4^{\circ} \mathrm{C}$ (mock shipping conditions) to mimic standard overnight shipping conditions. Each tissue lot must meet the Quality Control (QC) criteria which were first established in 2014. The purpose of the QC assay is to ensure reproducible tissue properties across independent tissue lots produced over time - an essential property for any toxicological test system (Rispin et al. 2006). The QC parameters were established based on QC data from 46 tissue lots produced during 2012-2013, following storage under mock shipping conditions.

The key parameter involved in EpiOral QC testing is the ET-50, which refers to the exposure time required for the reference chemical $(1.0 \%, \mathrm{v} / \mathrm{v})$ Triton $\mathrm{X}-100$, to reduce the tissue viability to $50 \%$, as measured by the MTT assay (Mosmann 1983; Klausner et al. 1997; Klausner et al.
2007). The MTT assay measures mitochondrial activity in the basal cells of the tissue. For a test material (such as Triton X-100) to decrease the MTT tissue viability, it must penetrate the epithelial barrier and impair normal mitochondrial function. Therefore, the MTT ET-50 indirectly monitors the barrier function of the tissue which is important for all topically applied materials. Recently, the EpiOral tissue has been shipped from the USA to Japan to service the Japanese oral care market. Due to extended shipping times and customs clearance, the tissue typically spends $48-72 \mathrm{~h}$ in the package. QC data for $n=23$ tissue lots tested at MatTek Corporation (Ashland, MA), $n=18$ lots tested at LION Corporation (Odawara, Japan), and $n=11$ lots tested at Kurabo Industries Ltd. (Osaka, Japan) are presented in Table 1. Although ET50s are slightly lower following the international shipment, all 
Table 1 Results of Quality Control (QC) testing.

\begin{tabular}{|c|c|c|c|c|c|c|c|}
\hline \multirow[b]{2}{*}{ Ship date } & \multirow[b]{2}{*}{ Lot } & \multicolumn{3}{|c|}{ ET-50 (min) } & \multicolumn{3}{|c|}{ NC OD average } \\
\hline & & MatTek & LION & Kurabo & MatTek & LION & Kurabo \\
\hline $10 / 23 / 17$ & 26173 & 90.8 & 61.8 & - & 1.559 & 1.242 & - \\
\hline $11 / 13 / 17$ & 26186 & 90.5 & 60.7 & - & 1.369 & 1.334 & - \\
\hline $1 / 15 / 18$ & 27712 & 90.1 & 75.7 & 45.7 & 1.564 & 1.739 & 1.789 \\
\hline $1 / 29 / 18$ & 27724 & 97.0 & 83.7 & 71.6 & 1.524 & 1.620 & 1.96 \\
\hline $2 / 26 / 18$ & 27739 & 80.8 & 64.6 & 71.1 & 1.523 & 1.710 & 1.803 \\
\hline $3 / 26 / 18$ & 27750 & 71.3 & 49.0 & - & 1.575 & 1.852 & - \\
\hline $4 / 9 / 18$ & 27753 & 89.2 & 59.1 & 56.6 & 1.489 & 1.675 & 1.803 \\
\hline $5 / 7 / 18$ & 27761 & 84.5 & 81.2 & - & 1.596 & 1.895 & - \\
\hline $5 / 21 / 18$ & 27762 & 77.8 & 53.0 & - & 1.450 & 1.627 & - \\
\hline $6 / 4 / 18$ & 27766 & 85.5 & 76.7 & - & 1.587 & 1.653 & - \\
\hline $6 / 25 / 18$ & 27772 & 73.0 & 71.5 & - & 1.606 & 1.673 & - \\
\hline $8 / 20 / 18$ & 27784 & 57.9 & 48.7 & 49.1 & 1.596 & 1.574 & 1.856 \\
\hline $9 / 17 / 18$ & 27793 & 94.2 & - & 45.5 & 1.580 & - & 2.033 \\
\hline $10 / 1 / 18$ & 27796 & 78.2 & 53.6 & - & 1.447 & 1.684 & - \\
\hline $10 / 15 / 18$ & 29500 & 88.8 & - & 57.4 & 1.406 & - & 1.703 \\
\hline $10 / 29 / 18$ & 29505 & 72.8 & 78.6 & - & 1.528 & 1.608 & - \\
\hline $11 / 12 / 18$ & 29508 & 96.9 & 79.8 & 63.6 & 1.623 & 1.501 & 1.724 \\
\hline $11 / 26 / 18$ & 29518 & 82.1 & 67.6 & - & 1.558 & 1.594 & - \\
\hline $12 / 10 / 18$ & 29524 & 48.6 & 45.5 & - & 1.696 & 1.684 & - \\
\hline $1 / 14 / 19$ & 29527 & 71.9 & - & 47.3 & 1.587 & - & 1.766 \\
\hline 2/11/19 & 29537 & 47.0 & 45.1 & - & 1.530 & 1.761 & - \\
\hline $6 / 3 / 19$ & 29564 & 85.5 & - & 42.0 & 1.577 & - & 1.773 \\
\hline \multirow[t]{4}{*}{$7 / 22 / 19$} & 29582 & 51.3 & - & 51.9 & 1.585 & - & 1.913 \\
\hline & Average & 78.2 & 64.2 & 54.7 & 1.545 & 1.635 & 1.829 \\
\hline & St dev & 14.2 & 12.8 & 10.3 & 0.076 & 0.154 & 0.102 \\
\hline & $N$ & 23 & 18 & 11 & & & \\
\hline \multirow[t]{2}{*}{ QC criteria: } & & \multicolumn{3}{|c|}{ PC: ET-50 } & \multicolumn{3}{|c|}{$\mathrm{NC}: \mathrm{OD}>1.0$} \\
\hline & & \multicolumn{6}{|c|}{$34.8 \mathrm{~min}<\mathrm{ET}-50<105.8 \mathrm{~min}$} \\
\hline
\end{tabular}

Results for standardized QC testing at MatTek (USA) after packaging and overnight storage at $4{ }^{\circ} \mathrm{C}$ or following 3-4 d international shipment to Kurabo Industries Ltd. (Osaka, Japan) and LION Corporation (Kanagawa, Japan). In Japan, QC testing was performed on day 3 following shipping except for two lots at LION and one lot at Kurabo in which testing was performed on day 4 due to weather-related shipping delays (delayed shipments are italicized in the table above). The ET-50 for the positive control (PC), $1 \%$ Triton X-100, and the optical density (OD) from the MTT assay for tissue exposed to the negative control (NC), ultrapure water, are shown. Although ET-50 values are slightly lower in Japan, all lots meet the QC acceptance criteria. The QC criteria listed were established based on data from 46 tissue lots produced during 2012-2013, following packaging and overnight storage at $4^{\circ} \mathrm{C}$. lots met the QC specifications when tested at LION and Kurabo. These results are similar to those for other mucosal models which also meet QC parameters following extended shipment times to Japan (Kaluzhny et al. 2006).

\section{Oral Irritation and Cytotoxicity}

Commercial producers of oral care products such as toothpastes, mouthwashes, and teeth whitening agents need a relatively simple, non-animal means of assessing the potential irritancy of their products when they are placed in the oral cavity. Using the MTT assay, researchers at MatTek and Procter and Gamble used the exposure time to decrease tissue viability to $50 \%$ (ET-50) to distinguish between the irritancy levels of toothpaste for adults, children, and infants. In addition, the release of the proinflammatory mediators interleukin$1 \alpha(\mathrm{IL}-1 \alpha)$ and IL-1 $\beta$ was measured to model the effect of common additives to oral care formulations on irritancy (Klausner et al. 2007). A group at Johnson \& Johnson used the EpiOral tissue model to investigate the effect of varying levels of ethanol in mouthwashes on tissue viability and 
transbuccal permeation. They found that common mouthwashes and solutions with ethanol content up to $26.9 \%$ had no effect on tissue viability or tissue morphology. In addition, these mouthwashes did not alter the transbuccal permeability of a model drug, caffeine (Koschier et al. 2011). Yang et al. 2011 used the EpiGingival tissue to study the retention of $o$ cymen-5-ol and zinc delivered by topical application of toothpaste. They found that significantly higher concentrations of these materials could be delivered using the toothpaste compared to equivalent doses delivered from solution. Importantly, no cytotoxic effects to the EpiGingival tissue were observed (Yang et al. 2011). Significantly, some groups have suggested that the 3D models are more relevant than $2 \mathrm{D}$ monolayer systems for cytotoxicity/biocompatibility studies (Moharamzadeh et al. 2008) and in one report, the EpiGingival was deemed the more appropriate model for evaluating the biocompatibility of bioadhesives (Moghaddam et al. 2016). In this study, lipophilic ingredients in the adhesive were toxic at all concentrations to monolayer cells but non-toxic to the EpiGingival tissue at the desired end-use concentration. The authors concluded that, given the ethical and regulatory restrictions related to animal studies for medical devices and cosmetic products, the 3D oral tissue models may be a more realistic and appropriate model for preclinical biocompatibility studies (Moghaddam et al. 2016).

In vitro tissue models of the oral mucosa have also been utilized to determine the irritancy properties of dental materials. EpiOral was used to ensure that chemical enhancers for iontophoresis did not disrupt tissue morphology ( $\mathrm{Hu}$ et al. 2010) and that a polymer blend to be used as a mucoadhesive was not toxic to the tissue (Song et al. 2017). The SkinEthic oral tissue was used to evaluate the toxicity of archwires used in orthodontic appliances. Vannet et al. compared archwires made of stainless steel (SS), a nickel-titanium alloy (Nitinol), or a titanium-molybdenum alloy (TMA) and determined their effects on tissue viability and histology. Of the three materials, the viability of the tissues exposed to the SS wire was indistinguishable from the negative control (non-exposed) tissues while the Nitinol and TMA wires reduced tissue viability by $15 \%$. Likewise, a comparison of histological cross-sections of tissues exposed to these wires showed the SS wire to be the most biocompatible (Vande Vannet et al. 2006). The same research group investigated different soldering methods and their effect on the SkinEthic HOE. Point welded (PW), laser welded (LW), and silver-soldered (SiS) were compared and although viability and histology effects were mild, the PW and LW wires induced less toxicity in terms of tissue viability and histology, when compared to the SiS wires (Vande Vannet and Hanssens 2007). In addition, this group found that non-cured orthodontic bonding adhesives caused cytotoxicity and caused histological changes to the HOE; effects were much milder for the polymerized adhesives (Vande Vannet et al. 2007).
The in vitro tissue models have also been used as an initial toxicity screen for novel materials that will be introduced into the oral cavity. Kovalchuk et al. 2013 studied new lipid antioxidants which were designed to kill breast cancer cells. They utilized the EpiOral and EpiGingival tissue models and did not see any effects on tissue morphology or gene expression when tissues were exposed to the lipid antioxidants (Kovalchuk et al. 2013). EpiOral and EpiGingival were also used to evaluate potential toxicity of silver nanoparticles (AgNP) which have advantageous antimicrobial properties. For exposure times up to $48 \mathrm{~h}$, researchers observed low toxicity and inflammatory effects in the AgNP-exposed tissues (Pindakova et al. 2017). Finally, Hayakumo et al. 2014 utilized EpiOral and EpiGingival to assess the cytotoxicity of a new antibacterial agent, ozone nano-bubble water (NBW3). They demonstrated rapid and potent bactericidal activity of the NBW3 against representative periodontopathogenic bacteria (e.g., $P$. gingivalis and A. actinomycetemcomitans) but did not observe any cytotoxicity to the oral tissue models after $24 \mathrm{~h}$ of exposure and suggested that NWB3 might be useful as an adjunct to current periodontal therapy (Hayakumo et al. 2014).

\section{Transbuccal Drug Delivery}

Due to its diminutive barrier properties, the buccal mucosa is an attractive site to administer drugs for either local or systemic delivery. For systemic delivery, direct access to the underlying capillaries and bloodstream would avoid the hepatic first pass metabolism and enzymatic degradation within the gastrointestinal tract (Senel and Hincal 2001; Smart 2005). Using the EpiOral tissue model, researchers have investigated the transbuccal delivery of small molecules such as nicotine for nicotine replacement therapy. Boateng et al. characterized the permeation of nicotine as part of smoking cessation therapy from hydroxypropyl methylcellulose/sodium alginate wafers and films (Boateng and Okeke 2019) and Battaglia and Nguyen 2017 showed that tincture of benzoin increased nicotine delivery by 2.1 -fold while preventing apoptosis (Battaglia and Nguyen 2017). The transbuccal delivery of macromolecules such as insulin has also been proposed. Studies of lyophilized chitosan xerogels loaded with insulin showed a linear relationship between insulin permeation in EpiOral tissue and in excised sheep buccal tissue (Giovino et al. 2013; Boateng et al. 2014). Another study focused on using permeation enhancers to deliver methylxanthines through the skin. In addition to transdermal data, researchers showed that delivery rates through the buccal tissue (EpiOral) were much higher and concluded that transbuccal delivery would offer an alternative means of delivery (Thakur et al. 2007). Using the SkinEthic HOE model, Nielsen et al. 1999 looked at the permeability of fluorescein isothiocyanate 
(FITC)-labeled dextrans (FD) over a range of molecular weights (MW) from 4000 to 40,000 . They found (a) that permeability of FD decreased as MW increased and (b) that the permeation enhancer, sodium glycocholate, was able to increase permeability rates up to a MW of 10,000 (Nielsen et al. 1999). Campisi et al. 2008 investigated the possibility of transbuccal delivery of carbamazepine (CBZ), an anticonvulsant drug. Even though $\mathrm{CBZ}$ readily permeated through the SkinEthic HOE tissue model, histological observations of the tissue showed disruption of the normal histological features of the oral mucosa. These observations were confirmed using porcine oral mucosa and although cytotoxicity was ruled out as the cause for these changes, the authors concluded that more studies would be necessary to assess the feasibility of transbuccal delivery of this drug (Campisi et al. 2008).

Researchers have also used oral tissue models to assess whether materials placed in the oral cavity would permeate through the buccal mucosa and thereby gain access to the circulatory system. Using the SkinEthic HOE model, Komiyama et al. 2019 studied nanomaterials used in the dental field. They found that nano-hydroxyapatite, a widely used synthetic form of the naturally occurring mineral found in tooth enamel and dentin, did not penetrate through the epithelium and thereby would likely not present any systemic toxicological issues (Komiyama et al. 2019). In a similar manner, based on the permeability of 5 -fluorouracil (5-FU), a drug currently used to treat oral squamous cell carcinoma (OSCC), through the HOE, it was concluded that there was low likelihood that 5-FU would enter the systemic circulatory system (Giannola et al. 2010).

\section{Fungal, Bacterial, and Viral Infection}

Candida albicans is a fungal organism that is typically part of the normal microbial flora in the oral cavity. However, C. albicans is polymorphic and can grow as hyphae, and in its hyphal form, it can invade the oral mucosa and cause tissue damage observed in oral candidiasis (also referred to as candidosis). C. albicans produced characteristic hallmarks of pathological tissue invasion in the SkinEthic HOE model over a period of $48 \mathrm{~h}$. Hyphae penetrated through epithelial cells and intercellular gaps later resembling thigmotropism (Jayatilake et al. 2008). Moyes et al. 2010 observed minimal expression of the mitogen-activated protein kinase phosphatase 1 (MKP1) and the c-Fos transcription factor in untreated HOE tissues. However, expression on the tissue surface was evident $4 \mathrm{~h}$ after infection with $C$. albicans and expression increased considerably $24 \mathrm{~h}$ post-infection (Moyes et al. 2010). Another group investigated the effect of HIV proteinase inhibitors and their effect on $C$. albicans infection of the HOE. They found that saquinavir reduced the tissue damage in the HOE and suggested it as a potential anti-candidal agent (Korting et al. 1999). Boros-Majewska utilized the SkinEthic HOE model to study the efficacy of novel derivatives of the antifungal antibiotic Nystatin A1 against $C$. albicans infection. One of the derivatives tested showed increased antifungal activity while showing lower toxicity compared to the uninfected HOE tissue (Boros-Majewska et al. 2014). Yadev et al. 2011 compared C. albicans infection of the SkinEthic HOE and MatTek EpiOral tissue models and looked at cytotoxicity, HBD2 expression, and the release of inflammatory cytokines such as IL- $1 \beta$, TNF- $\alpha$, and CXCL8 (IL-8) $24 \mathrm{~h}$ post-infection. Both tissues showed a similar cytotoxicity response as measured by lactate dehydrogenase (LDH) release but the expression of HBD2 increased only in the EpiOral tissue, similar to native oral mucosal tissue. In addition, the release of the inflammatory cytokines was much more pronounced in the EpiOral versus the SkinEthic tissue (Yadev et al. 2011). The authors concluded that EpiOral is a more advanced model of the oral mucosa and that it will likely aid in investigation of the molecular mechanisms involved in the innate immune responses to $C$. albicans infection.

In addition to infection studies with $C$. albicans, the oral tissue models have proven useful for studying other fungal and bacterial infections in the oral cavity. Silva et al. 2011 utilized the SkinEthic HOE to investigate infection with Candida tropicalis and demonstrated its ability to colonize the tissue. C. tropicalis was found to be highly invasive and induced significant damage within $24 \mathrm{~h}$ post-infection (Silva et al. 2011). Morse et al. 2018 utilized both the SkinEthic HOE and MatTek full thickness EpiOral tissue model to study the effects of various denture-associated biofilms including (a) fungal (C. albicans), (b) bacterial (Streptococcus sanguinis, S. gordini, Actinomyces viscosus, and A. odontolyticus), and (c) mixed species (created from C. albicans and the bacterial species listed in b). The biofilms induced cytotoxicity (LDH release), changes in gene expression, and histological effects in the tissues. While they found the models useful, they noted that the incorporation of immune cells to the models would expand their capabilities (Morse et al. 2018). The lack of an immunological component was addressed in a study by Brown et al. 2019 in which the SkinEthic HGE tissue model was cultured in the presence of immunological cells (peripheral blood mononuclear cells and CD34+ monocytes) and multi-species biofilms associated with healthy gingiva, gingivitis, and periodontitis. An inflammatory response in the immune cells was observed which was enhanced by the gingivitis-associated biofilm (Brown et al. 2019).

Various viral challenges to the oral cavity have been studied using the MatTek and SkinEthic oral tissue models. Exposure to HIV-1 did not upregulate the expression of HBD2 in the EpiOral tissue model even though modest increases were observed in monolayer cultures of gingival epithelial cells. Likewise, HIV-1 did not upregulate innate 
immune factors such as interferon regulatory factor 1 (IRF1), IL-1 $\beta$, chemokine ligand 5 (CCL5), and secretory leukocyte protease inhibitor (SPLI) even though it penetrated the upper layers of the tissue (Nittayananta et al. 2009). Human cytomegalovirus (HCMV), which causes oral diseases such as gingivitis, was shown to infect the EpiGingival tissue model. Use of EpiGingival as a HCMV infection model was demonstrated by (a) a dramatic increases in viral titer, (b) the production of viral proteins in infected tissues, and (c) the ability to inhibit viral growth by treating with ganciclovir, an antiviral drug which is used clinically to treat and prevent HCMV infection (Hai et al. 2006). In another very recent study, the angiotensin-converting enzyme II (ACE2), the key receptor for SARS-CoV-2 viral infection of cells (which leads to COVID-19), was found to be expressed in the EpiOral tissue. Extracts from Cannabis sativa were found to downregulate ACE2 expression, as well as the serine protease TMPRSS2, another critical protein required for SARS-CoV-2 entry into host cells. It was hypothesized that modulation of ACE2 levels in "gateway" tissues such as respiratory and oral mucosa may prove to be a plausible strategy for decreasing infection and disease susceptibility. As such, oral mucosal models have the potential to study potential SARS-CoV-2 infection in the oral cavity and to develop simple, easy-to-use preventative treatments (such as mouthwashes and throat gargle products) that could decrease infection and the susceptibility to COVID19 (Wang et al. 2020).

\section{Oral Pathology}

The painful inflammation and ulceration caused by oral mucositis can be a debilitating side effect of chemotherapy and radiation therapy for cancer patients. Lambros et al. irradiated the full thickness EpiOral tissue model with 2 and 12 Gray (Gy) of gamma irradiation to model oral mucositis and studied the effects on tissue morphology (histology), apoptosis, and gene expression, $6 \mathrm{~h}$ post-irradiation. The higher level of irradiation showed abnormal proliferation in histological crosssections and a significantly higher number of apoptotic cells versus the $2 \mathrm{~Gy}$ and the non-irradiated control tissues. In addition, the expression of several genes related to the NF-kB pathway and inflammatory cytokines, including IL- $1 \beta$, IL-8, NF-kB1, and FOS, was altered by irradiation (Lambros et al. 2011). In other related studies by the same group, prior to irradiation, EpiOral tissues were pre-treated with Qingre Liyan Decoction (QYD), a traditional Chinese medicine, and $N$-acetyl cysteine (NAC). Pre-treatment of the tissues reduced radiation damage effects, as evidenced by significant downregulation of apoptosis, cytokines, and chemokine genes, and constrained damage-associated molecular patterns or DAMPs (Lambros et al. 2015a; b).
The oral tissue models have also proven useful for investigating other phenomena of the oral activity which are linked to clinical pathologies. The importance of potassium ion transport and its connection to dysbiosis and periodontal disease was studied using the EpiGingival model. Potassium levels were associated with increased virulence of the oral microbiome while also altering the immune response of the tissue, as evidence by increased levels of TNF- $\alpha$ and decreased expression of IL-6 and the antimicrobial peptide human $\beta$-defensin-3 (Yost et al. 2017). In addition, the EpiGingival model was used to study the effects of biofilms as they relate to the expression of junctional proteins within the tissue and possible implications of periodontal disease (Belibasakis et al. 2015). Ramineni et al. 2015 investigated whether mucoadhesive films impregnated with epithelial growth factor (EGF) could be used to treat traumatic oral mucosal wounds. The mucoadhesive films delivered bioactive EGF in a sustained manner to punch biopsy wounds inflicted to the full thickness EpiOral tissue. However, the EGF treatment caused a hyperparakeratotic response and induced other structural abnormalities including thickening of the spinous layer, intra- and intercellular edema, and pyknotic nuclei. In addition, no improvements in wound closure were observed (Ramineni et al. 2015).

\section{Response to Tobacco Products}

In addition to the well-known effects of smoking related to lung cancer and chronic obstructive pulmonary disease (COPD), cigarette smoke is associated with cancer and inflammatory diseases of the oral cavity (Office of the Surgeon General US 2004). Using MatTek's full thickness EpiOral and EpiGingival tissues, researchers at Philip Morris International (Neuchatel, Switzerland) found that cigarette smoke increased the secretion of inflammatory cytokines and the activity of cytochrome P450 1A1 and 1B1, which have been shown to metabolize constituents of cigarette smoke (Port et al. 2004). Using microarrays and gene-set analysis, they showed induction of xenobiotic metabolismrelated pathways induced by cigarette smoke that were similar in the in vitro tissues to those seen in buccal biopsies from smokers (Schlage et al. 2014). The same group utilized EpiOral to evaluate an alternative, modified risk tobacco product which involves heating of tobacco, as opposed to the combustion thereof. They found that cytotoxicity, morphological changes, and the release of inflammatory mediators were all decreased using the alternate heating system when compared to cigarette smoke (Zanetti et al. 2016). The EpiGingival tissue was also used to investigate the effects of e-cigarettes and their flavorings on oral tissue health. Increased oxidative/ carbonyl stress and inflammatory cytokine release along with DNA damage were observed in the tissues. All of these effects 
were more pronounced for flavored e-cigarettes (Sundar et al. 2016). Thus, the oral tissue models are well suited to evaluate a variety of tobacco or alternative products in an effort to reduce adverse effects.

\section{Effects of Ultraviolet Radiation (UVR)}

The oral mucosa can be exposed to UV radiation as part of a diagnostic, therapeutic, dental, or cosmetic procedure as well as from direct sunlight. UVR induced the formation of cyclobutane pyrimidine dimers (CPD) and pyrimidine (6-4) pyrimidone photoproducts (6-4PP) in the EpiOral and EpiGingival tissue models. Although UV-induced damage was similar in these two tissue models and similar to that observed in a related skin model, the nucleotide excision repair rate for the oral cavity tissues was significantly below that of the skin tissue (Mitchell et al. 2012). In a related study, the number of apoptotic cells was decreased in the oral and gingival tissues versus the skin (Breger et al. 2013). The authors suggest that the use of UV in the oral cavity should be studied carefully since it could increase the risk of oral carcinoma or melanoma. The effectiveness of lip cream to protect against damaging effects of UVR was evaluated using the EpiGingival model. Unprotected tissue showed increases in tumor necrosis factor- $\alpha$ (TNF- $\alpha)$ and prostaglandin $E_{2}$ (PGE2) release following exposure to $150 \mathrm{~mJ} / \mathrm{cm}^{2}$ of UVB or $30 \mathrm{~J} / \mathrm{cm}^{2}$ of UVA. Application of a lip cream with a sun protection factor (SPF) of $\sim 12$ significantly decreased TNF- $\alpha$ and PGE2 levels following UV exposure, but levels were still higher than those of non-irradiated control tissues (Gfeller et al. 2019).

\section{Advantages, Disadvantages, and Limitations of 3D Culture Models}

When compared to $2 \mathrm{D}$ monolayer cultures, the $3 \mathrm{D}$ oral mucosal tissue models offer many advantages. Most importantly, the 3D models more accurately reproduce the structure, function, and underlying gene and protein expression of native mucosal tissues. These factors make the $3 \mathrm{D}$ models more appropriate for studying mucosal irritancy and cytotoxicity, transbuccal permeation, microbial infection, oral pathology, effects of tobacco use, and the effects of UV, among other phenomena affecting the oral cavity, as presented in this paper. Despite these advantages of 3D tissue models, 2D systems are much less expensive and more amenable to highthroughput screening and hence are useful for initial studies related to drug screening or studying isolated reactions or pathways.

Versus animal models, 3D tissue models (cultured utilizing human cells) have several advantages including no species extrapolation, enhanced reproducibility, and the avoidance of ethical issues related to using laboratory animals. Culture models are not subject to the restrictions placed on animal testing of toothpastes and other oral care products, which cannot be sold in the European Union if they have been tested on animals (Regulation EC No 1223/2009). In addition, 3D tissue models allow researchers to study isolated phenomena without complicating factors of complex in vivo systems. For instance, transbuccal drug delivery studies are straightforward using the 3D models while obtaining blood samples and the possibility of enzymatic degradation of the drug in the bloodstream of an animal model are complicating factors. Nonetheless, the commercial 3D models lack the complexity present in in vivo systems. Immune cells are lacking, with the exception of a single EpiOral tissue study (Schlage et al. 2014) and a single SkinEthic HGE study (Brown et al. 2019). Likewise, the commercial 3D models lack a vasculature, although an academic model has reported progress in this area (Nishiyama et al. 2019).

$3 \mathrm{D}$ oral mucosal organoids are another 3D system that has been developed for investigation of oral tissue regeneration or carcinogenesis (Hisha et al. 2013). In one study, tumor cells from the oral cavity (floor of mouth, tongue, and gingiva) were removed from patients and cultured to form $3 \mathrm{D}$ spherical organoids (diameter range: $100-300 \mu \mathrm{m}$ ) which recapitulated the genetic, molecular, and functional characteristics of the tumors (Driehuis et al. 2019). These organoids could find utility as a platform for drug screening but would not readily be amenable to transbuccal permeability measurements or other applications in which the polarity of the $3 \mathrm{D}$ tissue models is important.

A comprehensive comparison of the commercial 3D oral mucosal tissue models has not been reported. Regarding the gingival tissues, the SkinEthic HGE and MatTek EpiGingival tissue models are similar. Both models are cultured using normal human gingival cells and they adopt a stratified, cornified morphology. They have proliferative basal cells, similar to native gingival tissue, and express similar markers of differentiation (https://www.episkin.com/HGE-GingivalEpithelium; Kimball et al. 2006; Yadev et al. 2011). Regarding the buccal tissues, the main difference between the SkinEthic HOE and MatTek EpiOral tissue models relates to the cells used. The HOE model is cultured using the TR146 buccal carcinoma-derived cell line, while the EpiOral tissue is cultured using oral keratinocytes harvested from normal, non-cancerous tissue. The two models have been used to study similar phenomena affecting the oral cavity such as the biocompatibility of dental materials, the transbuccal permeation, and the effects of microbial infection of the tissues. In one study, the models were directly compared for their utility in developing a model of oral candidiasis. The EpiOral tissues were shown to more closely mimic the proliferation index of native tissue and they expressed innate 
immune molecules which mimicked the pattern observed for normal oral mucosa. Cytokine release patterns were also different following infection for the two models. The authors of this study concluded that EpiOral model represents a more advanced model for the oral mucosa which should prove useful in determining the innate immune response against $C$. albicans (Yadev et al. 2011). It is not clear whether the differences between the models would have implications for other applications as well. However, it is generally accepted that results using models based on cell lines should be interpreted carefully, since cell lines may not accurately reproduce properties or responses of normal epithelial cells (DongariBagtzoglou and Kashleva 2006).

\section{Conclusions}

As presented in this review, commercially available tissue models of the human buccal and gingival mucosa have been used to study a broad variety of phenomena in the oral cavity. These models offer a reproducible, non-animal means of isolating the effects of oral care products, smoking, ultraviolet radiation, radiation treatment, or dental materials on the oral cavity tissues. In addition, microbial infection models have been developed to study pathological conditions within the oral cavity. In most instances, the oral tissue models offer an effective means of studying the phenomenon of interest without species extrapolation or the ethical issues related to human or animal experimentation.

Acknowledgments The authors thank Jennifer Molignano of MatTek Corporation for reviewing and editing the manuscript. The EpiOral and EpiGingival tissues were originally developed using funds from SBIR grant \#R44 DE013277 from the National Institute of Dental and Craniofacial Research of the National Institutes of Health (Bethesda, MD).

\section{References}

Battaglia A, Nguyen T (2017) Transmucosal delivery of nicotine in combination with tincture of benzoin inhibits apoptosis. Drugs R D 17: 615-621

Belibasakis GN, Kast JI, Thurnheer T, Akdis CA, Bostanci N (2015) The expression of gingival epithelial junctions in response to subgingival biofilms. Virulence. 6(7):704-709

Benson K, Cramer S, Galla HJ (2013) Impedance-based cell monitoring: barrier properties and beyond. Fluids Barriers CNS 10(1):5

Bierbaumer L, Schwarze UY, Gruber R, Neuhaus W (2018) Cell culture models of oral mucosal barriers: a review with a focus on applications, culture conditions and barrier properties. Tissue Barriers 6(3): 1479568

Boateng JS, Mitchell JC, Pawar H, Ayensu I (2014) Functional characterisation and permeation studies of lyophilised thiolated chitosan xerogels for buccal delivery of insulin. Protein Pept Lett 21(11): $1163-1175$
Boateng JS, Okeke O (2019) Evaluation of clay-functionalized wafers and films for nicotine replacement therapy via buccal mucosa. Pharmaceutics 11:104

Boros-Majewska J, Salewska N, Borowski E, Milewski S, Malic S, Wei XQ, Hayes AJ, Wilson MJ, Williams DW (2014) Novel Nystatin $A_{1}$ derivatives exhibiting low host cell toxicity and antifungal activity in an in vitro model of oral candidosis. Med Microbiol Immunol 203(5):341-355

Breger J, Baeva L, Agrawal A, Shindell E, Godar DE (2013) UVBinduced inflammatory cytokine release, DNA damage and apoptosis of human oral compared with skin tissue equivalents. Photochem Photobiol 89(3):665-670

Brown JL, Johnston W, Delaney C, Rajendran R, Butcher J, Khan S, Bradshaw D, Ramage G, Culshaw S (2019) Biofilm-stimulated epithelium modulates the inflammatory responses in co-cultured immune cells. Sci Rep 9:15779. https://doi.org/10.1038/s41598-01952115-7

Campisi G, Paderni C, Saccone R, Siragusa MG, Lo Muzio L, Tripodo C, Giannola LI, Florena AM (2008) Carbamazepine transbuccal delivery: the histo-morphological features of reconstituted human oral epithelium and buccal porcine mucosae in the transmucosal permeation. Int J Immunopathol Pharmacol 21(4):903-910

Delcourt-Huard A, Corlu A, Joffre A, Magloire H, Bonnaure-Mallet M (1997) Reconstituted human gingival epithelium: nonsubmerged in vitro model. In Vitro Cell Dev Biol Anim 33(1):30-36

Dongari-Bagtzoglou A, Kashleva H (2006) Development of a highly reproducible three-dimensional organotypic model of the oral mucosa. Nat Protoc 1(4):2012-2018

Driehuis E, Kolders S, Spelier S, Lõhmussaar K, Willems SM, Devriese LA, de Bree R, de Ruiter EJ, Korving J, Begthel H, van Es JH, Geurts V, He G-W, van Jaarsveld RH, Oka R, Muraro MJ, Vivié J, Zandvliet MMJM, Hendrickx APA, Iakobachvili N, Sridevi P, Kranenburg O, van Boxtel R, Kops GJPL, Tuveson DA, Peters PJ, van Oudenaarden A, Clevers H (2019) Oral mucosal organoids as a potential platform for personalized cancer therapy. Cancer Discov 7:852-871

Gfeller CF, Wanser R, Mahalingam H, Moore DJ, Wang X, Lin CB, Shanga G, Grove G, Rawlings AV (2019) A series of in vitro and human studies of a novel lip cream formulation for protecting against environmental triggers of recurrent Herpes labialis. Clin Cosmet Investig Dermatol 22(12):193-208

Giannola LI, De Caro V, Giandalia G, Siragusa MG, Paderni C, Campisi G, Florena AM (2010) 5-Fluorouracil buccal tablets for locoregional chemotherapy of oral squamous cell carcinoma: formulation, drug release and histological effects on reconstituted human oral epithelium and porcine buccal mucosa. Curr Drug Deliv 7(2):109-117

Giovino C, Ayensu I, Tetteh J, Boateng JS (2013) An integrated buccal delivery system combining chitosan films impregnated with peptide loaded PEG-b-PLA nanoparticles. Colloids Surf B: Biointerfaces 112:9-15

Hai R, Chu A, Li H, Umamoto S, Rider P, Liu F (2006) Infection of human cytomegalovirus in cultured human gingival tissue. Virol J 3: 84

Hayakumo S, Arakawa S, Takahashi M, Kondo K, Mano Y, Izumi Y (2014) Effects of ozone nano-bubble water on periodontopathic bacteria and oral cells -in vitro studies. Sci Technol Adv Mater 15(5):055003

Hisha H, Tanaka T, Kanno S, Tokuyama Y, Komai Y, Ohe S, Yanai H, Omachi T, Ueno H (2013) Establishment of a novel lingual organoid culture system: generation of organoids having mature keratinized epithelium from adult epithelial stem cells. Sci Rep 3: 3224

Hu L, Damaj BB, Martin R, Michniak-Kohn BB (2010) Enhanced in vitro transbuccal drug delivery of ondansetron HCl. Int J Pharm 404(12):66-74 
Jacobsen J, van Deurs B, Pedersen M, Romer Rassing M (1995) TR146 cells grown on filters as a model for human buccal epithelium: I. morphology, growth, barrier properties, and permeability. Int $\mathrm{J}$ Pharm 125:165-184

Jayatilake JAMS, Samaranayake YH, Samaranayake LPA (2008) Comparative study of candidal invasion in rabbit tongue mucosal explants and reconstituted human oral epithelium. Mycopathologia 165:373-380

Jensen C, Teng Y (2020) Is it time to start transitioning from 2D to 3D cell culture? Front Mol Biosci 7:33

Kaluzhny Y, Kandárová H, Handa Y, Deluca J, Truong T, Hunter A, Kearney P, d'Argembeau L, Klausner M (2006) The EpiOcular Eye Irritation Test (EIT) for hazard identification and labelling of eye irritating chemicals: protocol optimisation for solid materials and the results after extended shipment. ATLA 43:101-127

Kimball JR, Nittayananta W, Klausner M, Chung WO, Dale BA (2006) Antimicrobial barrier of an in vitro oral epithelial model. Arch Oral Biol 51:775-783

Klausner M, Ayehunie S, Breyfogle BA, Wertz PW, Bacca L, Kubilus J (2007) Organotypic human oral tissue models for toxicological studies. Toxicol in Vitro 21:938-949

Klausner M, Neal PJ, Cannon CL, Kubilus J, Curren RD, Harbell JW (1997) Intra- and interlaboratory reproducibility of EpiDerm, an in vitro model for dermal irritancy testing. In: Salem $\mathrm{H}$, Katz SA (eds) Advances in animal alternatives. Taylor \& Francis, Washington, DC, pp 347-357

Komiyama S, Miyasaka R, Kikukawa K, Hayman R (2019) Can nanohydroxyapatite permeate the oral mucosa? A histological study using three-dimensional tissue models. PLoS One 14(4)

Korting HC, Schaller M, Eder G, Hamm G, Böhmer U, Hube B (1999) Effects of the human immunodeficiency virus (HIV) proteinase inhibitors saquinavir and indinavir on in vitro activities of secreted aspartyl proteinases of Candida albicans isolates from HIVinfected patients. Antimicrob Agents Chemother 43(8):2038-2042

Koschier F, Kostrubsky V, Toole C, Gallo MA (2011) In vitro effects of ethanol and mouthrinse on permeability in an oral buccal mucosal tissue construct. Food Chem Toxicol 49(10):2524-2929

Kovalchuk A, Aladedunye F, Rodriguez-Juarez R, Li D, Thomas J, Kovalchuk O, Przybylski R (2013) Novel antioxidants are not toxic to normal tissues but effectively kill cancer cells. Cancer Biol Ther 14:907-915

Lambros MP, DeSalvo MK, Moreno J, Mulamalla HC, Kondapalli L (2015a) Transcriptional profiling of radiation damage and preventive treatments in a 3-dimensional (3D) human cell culture model of oral mucositis. Genomics Data 6:40-43

Lambros MP, Kondapalli L, Parsa C, Mulamalla HC, Orlando R, Pon D, Huang Y, Chow MSS (2015b) Signatures in the prevention of radiation damage by the synergistic effect of $\mathrm{N}$-acetyl cysteine and Qingre Liyan Decoction, a traditional Chinese medicine, using a 3-dimensional cell culture model of oral mucositis. Evid Based Complement Altern Med 2015:Article ID 425760. https://doi.org/ $10.1155 / 2015 / 425760$

Lambros MP, Parsa C, Mulamalla HC, Orlando R, Lau B, Huang Y, Pon D, Chow M (2011) Identifying cell and molecular stress after radiation in a three-dimensional (3-d) model of oral mucositis. Biochem Biophys Res Comm 405:102-106

Mitchell D, Paniker L, Godar D (2012) Nucleotide excision repair is reduced in oral epithelial tissues compared with skin. Photochem Photobiol 88:1027-1032

Moghaddam B, Yang J, Roohpour N (2016) Biologic evaluation of devices with chronic exposure using $3 \mathrm{D}$ human gingival model. Front Bioeng Biotech 4. https://doi.org/10.3389/conf.FBIOE.2016.01. 01697

Moharamzadeh K, Brook IM, Scutt AM, Thornhill MH, Van Noort R (2008) Mucotoxicity of dental composite resins on a tissue- engineered human oral mucosal model. J Dent 36(5):331-336. https://doi.org/10.1016/j.jdent.2008.01.019

Morse DJ, Wilson MJ, Wei X, Lewis MAO, Bradshaw DJ, Murdoch C, Williams DW (2018) Denture-associated biofilm infection in threedimensional oral mucosal tissue models. J Med Microbiol 67:364375

Mosmann T (1983) Rapid colorimetric assay for cellular growth and survival: application to proliferation and cytotoxicity assays. J Immunol Methods 65:55-63

Moyes DL, Runglall M, Murciano C, Shen C, Nayar D, Thavaraj S, Kohli A, Islam A, Mora-Montes H, Challacombe SJ, Naglik JR (2010) A biphasic innate immune MAPK response discriminates between the yeast and hyphal forms of Candida albicans in epithelial cells. Cell Host Microbe 8(3):225-235

Nielsen HM, Verhoef JC, Ponec M, Rassing MR (1999) TR146 cells grown on filters as a model of human buccal epithelium: permeability of fluorescein isothiocyanate-labelled dextrans in the presence of sodium glycocholate. J Control Release 60(2-3):223-233

Nishiyama K, Akagi T, Iwai S, Akashi M (2019) Construction of vascularized oral mucosa equivalents using a layer-by-layer cell coating technology. Tissue Eng C: Methods:262-275

Nittayananta W, Hladik F, Klausner M, Harb S, Dale W (2009) HIV type 1 fails to trigger innate immune factor synthesis in differentiated oral epithelium. AIDS Res Hum Retrovir 25:1013-1021

Office of the Surgeon General US (2004) The health consequences of smoking: a report of the surgeon general. Atlanta: Centers for Disease Control and Prevention)

Pindakova L, Kasparkova V, Kejlova K, Dvorakova M, Krsek D, Jirova D, Kasparova L (2017) Behaviour of silver nanoparticles in simulated saliva and gastrointestinal fluids. Int J Pharm 527:12-20

Port JL, Yamaguchi K, Du B, De Lorenzo M, Chang M, Heerdt PM, Kopelovich L, Marcus CB, Altorki NK, Subbaramaiah K, Dannenberg AJ (2004) Tobacco smoke induces CYP1B1 in the aerodigestive tract. Carcinogenesis 25:2275-2281

Ramineni SK, Fowler CB, Fisher PD, Cunningham LL, Puleo DA (2015) Effects of epidermal growth factor loaded mucoadhesive films on wounded oral tissue rafts. Biomed Mater 10:015026. https://doi.org/ 10.1088/1748-6041/10/1/015026

REGULATION (EC) No 1223/2009 of the European Parliament and of the council of 30 November 2009 on cosmetic products. https://eurlex.europa.eu/legal-content/EN/TXT/HTML/?uri=CELEX: 02009R1223-20150416\&from=EN

Rispin A, Stitzel K, Harbell J, Klausner M (2006) Ensuring quality of in vitro alternative test methods: current practice. Regul Toxicol Pharmacol 45:97-103

Rupniak HT, Rowlatt C, Lane EB, Steele JG, Trejdosiewicz LK, Laskiewicz B, Povey S, Hill BT (1985) Characteristics of four new human cell lines derived from squamous cell carcinomas of the head and neck. J Natl Cancer Inst 75(4):621-635

Sa G, Xiong X, Wu T, Yang J, He S, Zhao Y (2016) Histological features of oral epithelium in seven animal species: as a reference for selecting animal models. Eur J Pharm Sci 81:10-17

Samaranayake YH, Samaranayake LP (2001) Experimental oral candidiasis in animal models. Clin Microbiol Rev 14:398-429

Schlage WK, Iskandar AR, Kostadinova R, Xiang Y, Sewer A, Majeed S, Kuehn D, Frentzel S, Talikka M, Geertz M, Mathis C, Ivanov N, Hoeng J, Peitsch MC (2014) In vitro systems toxicology approach to investigate the effects of repeated cigarette smoke exposure on human buccal and gingival organotypic epithelial tissue cultures. Toxicol Mech Methods 24(7):470-487

Senel S, Hincal AA (2001) Drug permeation enhancement via buccal route: possibilities and limitations. J Control Release 72(1-3):133144

Silva S, Hooper SJ, Henriques M, Oliveira R, Azeredo J, Williams DW (2011) The role of secreted aspartyl proteinases in Candida tropicalis 
invasion and damage of oral mucosa. Clin Microbiol Infect 17(2): 264-272

Smart JD (2005) Buccal drug delivery. Expert Opin Drug Deliv 2(3): $507-517$

Song G, Banov D, Bassani AS, Valdez BC (2017) Evaluation of the safety, cell migration, and mucoadhesive properties of a mucoadhesive polymer blend in human oral mucosa. AAPS PharmSciTech 18:1617-1623

Squier CA, Kremer MJ (2001) Biology of oral mucosa and esophagus. J Natl Cancer Inst Monogr 29:7-15

Sundar IK, Javed F, Romanos GE, Rahman I (2016) E-cigarettes and flavorings induce inflammatory and prosenescence responses in oral epithelial cells and periodontal fibroblasts. Oncotarget 7(47):7719677204

Thakur RA, Michniak BB, Meidan VM (2007) Transdermal and buccal delivery of methylxanthines through human tissue in vitro. Drug Dev Ind Pharm 33:513-521

Vande Vannet B, Hanssens JL, Wehrbein H (2007) The use of threedimensional oral mucosa cell cultures to assess the toxicity of soldered and welded wires. Eur J Orthod 29(1):60-66

Vande Vannet B, Mohebbian N, Wehrbein H (2006) Toxicity of used orthodontic archwires assessed by three-dimensional cell culture. Eur J Orthod 28(5):426-432
Vande Vannet BMRA, Hanssens JL (2007) Cytotoxicity of two bonding adhesives assessed by three-dimensional cell culture. Angle Orthod 77(4):716-722

Wang B, Kovalchuk A, Li D, Inytskyy Y, Kovalchuk I, Kovalchuk O (2020) In search of preventative strategies: novel anti-inflammatory high-CBD cannabis sativa extracts modulate ACE2 expression in COVID-19 gateway tissues. Preprints 2020:2020040315. https:// doi.org/10.20944/preprints202004.0315.v1

Yadev NP, Murdoch C, Saville SP, Thornhill MH (2011) Evaluation of tissue engineered models of the oral mucosa to investigate oral candidiasis. Microb Pathog 50:278-285

Yang J, Deol G, Myangar N (2011) Retention of o-cymen-5-ol and zinc on reconstructed gingival tissue from a toothpaste formulation. Int Dentral J 61(Suppl 3):41-45

Yost S, Duran-Pinedo AE, Krishnan K, Frias-Lopez J (2017) Potassium is a key signal in host-microbiome dysbiosis in periodontitis. PLoS Pathog 13(6):e1006457

Zanetti F, Sewer A, Mathis C, Iskandar AR, Kostadinova R, Schlage WK, Leroy P, Majeed S, Guedj E, Trivedi K, Martin F, Elamin A, Merg C, Ivanov NV, Frentzel S, Peitsch MC, Hoeng J (2016) Systems toxicology assessment of the biological impact of a candidate modified risk tobacco product on human organotypic oral epithelial cultures. Chem Res Toxicol 29:1252-1269 